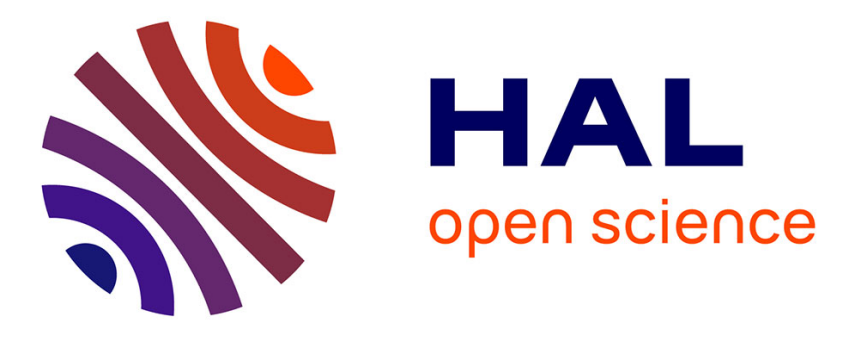

\title{
A hybrid control framework for impulsive control of satellite rendezvous
}

Mirko Brentari, Denis Arzelier, Christophe Louembet, Laura Sofia Urbina, Luca Zaccarian

\section{- To cite this version:}

Mirko Brentari, Denis Arzelier, Christophe Louembet, Laura Sofia Urbina, Luca Zaccarian. A hybrid control framework for impulsive control of satellite rendezvous. 2016 American Control Conference, Jul 2016, Boston, United States. hal-01214097v2

\section{HAL Id: hal-01214097 \\ https://hal.science/hal-01214097v2}

Submitted on 12 Apr 2016

HAL is a multi-disciplinary open access archive for the deposit and dissemination of scientific research documents, whether they are published or not. The documents may come from teaching and research institutions in France or abroad, or from public or private research centers.
L'archive ouverte pluridisciplinaire HAL, est destinée au dépôt et à la diffusion de documents scientifiques de niveau recherche, publiés ou non, émanant des établissements d'enseignement et de recherche français ou étrangers, des laboratoires publics ou privés. 


\title{
A hybrid control framework for impulsive control of satellite rendezvous
}

\author{
Mirko Brentari, Denis Arzelier, Christophe Louembet, Sofia Urbina and Luca Zaccarian
}

\begin{abstract}
We focus on the problem of satellite rendezvous between two spacecraft in elliptic orbits. Using a linearized model of the relative dynamics, we first propose a periodic similarity transformation based on Floquet-Lyapunov theory, leading to a set of coordinates under which the free motion is linear time-invariant. Then we address the problem of impulsive control of satellite rendezvous as a hybrid dynamical system, and we show that the arising elegant representation enables designing impulsive control laws with different tradeoffs between computational complexity and fuel consumption. The adopted hybrid formalism allows us to prove suitable stability properties of the proposed controllers. The results are comparatively illustrated on simulation examples.
\end{abstract}

\section{INTRODUCTION}

Considering the increasing need for satellite servicing in space, the capability of operating an active spacecraft, the follower denoted by $\mathrm{F}$, in close proximity of a satellite, the leader denoted by $\mathrm{L}$ will be crucial for fulfilling complex safe space missions objectives comprising inspection, repairing, refueling or monitoring [5]. The whole relative spacecraft maneuvering process composes what is known as the rendezvous and proximity operations which mainly consists in getting the follower from one orbit to a box near the leader (close range rendezvous) [9], [4] and then in beginning the proximity operations required by the mission objectives. When dealing with the preliminary planning phase of space missions, it is usual to approximate actual finite-thrust powered phases of finite duration by impulsive maneuvers. The impulsive approximation for the thrust means that instantaneous velocity increments are applied to the chaser when firing, whereas its position is continuous. This assumption, made in this paper, has proved to be very useful in reducing the complexity of guidance and control design and has been widely used in the literature dedicated to rendezvous (see [5], [3], [4] and references therein).

In this article, we are mainly interested by the first phase for which it is highly recommended to design fuel efficient impulsive maneuvers guiding the follower, from one point to a specified tolerance region in the proximity of the leader where the relative motion of the follower will be periodic and bounded. It is well known that, under Keplerian assumptions, the relative motion between spacecraft is bounded [7]. Different conditions for the periodicity of the linearized equations of the nonlinear relative motion have been given in the literature. Here we consider the parametric expression for the relative motion proposed in [3] and used to characterize in a simple way periodic relative motions. In a linearized context, any relative periodic trajectory is defined by 6 constant parameters, one of them being equal to 0 . In particular,

\footnotetext{
D. Arzelier, C.
Louembet,
Université de $\begin{aligned} & \text { Urbina } \\ & \text { Toulouse, }\end{aligned}$ CNRS, France arzelier, louembet, lsurbina, zaccarian@laas.fr

M. Brentari and L. Zaccarian are with Dipartimento di Ingegneria Industriale, University of Trento, Italy

Work supported in part by ANR under project LimICoS, contract number 12 BS03 005 01, by the iCODE institute, research project of the Idex Paris-Saclay, and by grant OptHySYS funded by the University of Trento.

Work supported in part by CNES under convention 117826.
}

building on the result presented in [3], the contribution of the present paper is twofold. First, we propose a new coordinate transformation which leads to a simplified characterization of periodic trajectories when applied to the Tschauner-Hempel equations of the elliptic linearized relative motion. Second, three different hybrid feedback-control laws are designed by taking advantage of the particular formulation of the rendezvous problem. The use of the hybrid framework [6] for representing nonlinear hybrid dynamical systems (whose solutions exhibit continuous evolution and impulsive behavior) allows us to state and prove suitable stability properties of the proposed impulsive control laws (including, e.g., the one originally presented in [4]) when applied to the linear timevarying dynamics of the closed-loop satellite rendezvous system. Due to lack of space, all statements and proofs are omitted but are given in the extended version [2].

Notations: $e, \nu$ and $T$ are respectively the eccentricity, the true anomaly and the period of the leader's orbit. $f^{\prime}$ represents the derivation of the function $f$ with respect to the true anomaly $\nu . I_{n}$ is the identity matrix of dimension $n$. For a set $S, \bar{S}$ denotes the closure of the set $S$.

\section{LTI STATE-SPACE FOR THE LINEARISED RELATIVE EQUATIONS OF MOTION}

The proximity operations between two spacecraft are characterized by the use of relative navigation since the separation between spacecraft is sufficiently small. In this framework, the relative motion of the follower is described in the Local-Vertical-Local-Horizontal (LVLH) frame attached to the leader body [9]. The origin of the coordinate frame is located at the center of mass of the leader and the space is spanned by $(x, y, z)$ where the $z$ axis is in the radial direction (R-bar) oriented towards the center of the Earth, the $y$ axis is perpendicular to the leader orbital plane and pointing in the opposite direction of the angular momentum (H-bar) while the $x$ axis is chosen such that $x=y \times z$.

Under Keplerian assumptions (no orbital perturbations are considered) and an elliptic reference orbit, the equations of motion for the relative motion in the LVLH frame may be linearized for close separation between the leader and the follower and are known as the Tschauner-Hempel (TH) equations [12], [11]:

$$
\begin{array}{lc}
\dot{\zeta}=A_{0}(t) \zeta & \text { free motion } \\
\zeta^{+}=\zeta+\left[\begin{array}{c}
0_{3 \times 3} \\
I_{3}
\end{array}\right] \int_{t^{-}}^{t^{+}} \frac{f(t)}{m_{F}} d t & \begin{array}{c}
\text { when applying } \\
\text { impulsive thrusts, }
\end{array}
\end{array}
$$

where state $\zeta=(x, y, z, d x / d t, d y / d t, d z / d t)$ represents positions and velocities in the three fundamental axes of the LVLH frame, matrix $A_{0}$ is a suitable periodic function of time $t[12], f(t)$ is the thrust vector, $\zeta^{+}$is the state vector right after the jump and $m_{F}$ is the mass of the follower. We may define the impulsive control input (essentially equivalent 
to velocity jumps in the three axes) as:

$$
\Delta v\left(t_{k}\right):=\int_{t_{k}^{-}}^{t_{k}^{+}} \frac{1}{m_{F}}\left[\begin{array}{l}
f_{x}(t) \\
f_{y}(t) \\
f_{z}(t)
\end{array}\right] d t
$$

where $t_{k}$ is a generic firing time, which is directly associated to the fuel consumption.

In order to simplify the $\mathrm{TH}$ equations, classical derivations correspond to applying a change of independent variable from time $t$ to true anomaly $\nu$, noting that:

$$
\frac{d \nu}{d t}=\frac{n}{\left(1-e^{2}\right)^{3 / 2}}(\underbrace{1+e \cos \nu}_{\rho(\nu)})^{2}=: k^{2} \rho(\nu)^{2},
$$

where $n=2 \pi / T$ is the mean motion of the leader orbit, satisfying for any fixed $\nu_{0}, t_{0}$ :

$$
\nu-\nu_{0}=2 \pi \quad \Rightarrow \quad n\left(t-t_{0}\right)=2 \pi \text {. }
$$

This leads to the following hybrid representation for a new state $\xi(\nu)$, replacing $\zeta(t)$ :

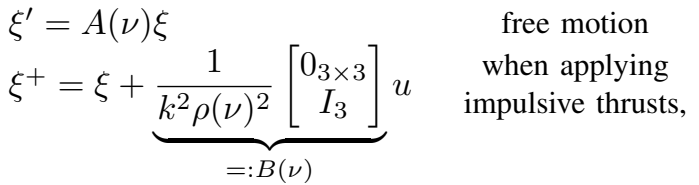

where $u=\Delta v$ represents the applied impulse, state $\xi=(x, y, z, d x / d \nu, d y / d \nu, d z / d \nu)$ represents positions and velocities with respect to $\nu$, and $A(\nu):=$ $\left[\begin{array}{cccccc}0 & 0 & 0 & 1 & 0 & 0 \\ 0 & 0 & 0 & 0 & 1 & 0 \\ 0 & 0 & 0 & 0 & 0 & 1 \\ \frac{e c_{\nu}}{\rho} & 0 & -2 \frac{e s_{\nu}}{\rho} & 2 \frac{e s_{\nu}}{\rho} & 0 & 2 \\ 0 & -\frac{1}{\rho} & 0 & 0 & 2 \frac{e s_{\nu}}{\rho} & 0 \\ 2 \frac{e s_{\nu}}{\rho} & 0 & \frac{2+\rho}{\rho} & -2 & 0 & 2 \frac{e s_{\nu}}{\rho}\end{array}\right]$ (see [12], [11]), where the dependence on $\nu$ has been omitted for brevity's sake and where we used $c_{\nu}:=\cos (\nu)$ and $s_{\nu}:=\sin (\nu)$.

Previous work [12], showed the usefulness of the following coordinate change for simplifying expression of dynamics (5):

$$
T(\nu):=\left[\begin{array}{cc}
\rho(\nu) I_{3 \times 3} & 0_{3 \times 3} \\
\rho(\nu)^{\prime} I_{3 \times 3} & \rho(\nu) I_{3 \times 3}
\end{array}\right] .
$$

In this article, we propose the two following additional transformations, the first one arising from similar observations to those in [4], and the second one arising from a suitable Floquet-Lyapunov derivation:

$$
\begin{aligned}
& C(\nu):= \\
& {\left[\begin{array}{cccccc}
0 & c_{\nu} & 0 & 0 & -s_{\nu} & 0 \\
0 & s_{\nu} & 0 & 0 & c_{\nu} & 0 \\
1 & 0 & -\frac{3 e s_{\nu}(1+\rho)}{\rho\left(e^{2}-1\right)} & \frac{e s_{\nu}(1+\rho)}{e^{2}-1} & 0 & \frac{\rho^{2}-e c_{\nu}-3}{e^{2}-1} \\
e & 0 & -3 s_{\nu} & s_{\nu}(1+\rho) & 0 & c_{\nu} \rho \\
0 & 0 & \frac{3\left(c_{\nu}+e\right)}{e^{2}-1} & -\frac{c_{\nu}(1+\rho)+e}{e^{2}-1} & 0 & s_{\nu} \rho \\
0 & 0 & -\frac{3\left(3 e c_{\nu}+e^{2}+2\right)}{e^{2}-1} & \frac{3 \rho^{2}}{e^{2}-1} & 0 & -\frac{3 e s_{\nu} \rho}{e^{2}-1}
\end{array}\right]} \\
& S(\nu):=\left[\begin{array}{cc|cccc}
1 & 0 & 0 & 0 & 0 & 0 \\
0 & 1 & 0 & 0 & 0 & 0 \\
\hline 0 & 0 & 1 & 0 & 0 & \frac{\sigma(\nu)}{\left(1-e^{2}\right)^{3 / 2}} \\
0 & 0 & 0 & 1 & 0 & 0 \\
0 & 0 & 0 & 0 & 1 & 0 \\
0 & 0 & 0 & 0 & 0 & 1
\end{array}\right],
\end{aligned}
$$

where we introduced the function:

$$
\begin{aligned}
\sigma(\nu) & :=(\nu-\tilde{\nu})-n(t-\tilde{t}) \\
& =(\nu-\tilde{\nu})-(M-\tilde{M}) \\
& =\Delta \nu-\Delta M,
\end{aligned}
$$

based on an arbitrary true anomaly value $\tilde{\nu}$ (in our simulations we select $\tilde{\nu}=0$ ) corresponding to a specific time value $\tilde{t}$ (in our simulations we select $\tilde{t}=0$ ). Function $\sigma$ in (9) is clearly periodic and bounded, due to the geometric dependence of $\nu$ and $M$ when the leader follows a Keplerian elliptic motion.

The following result establishes a first contribution of this paper showing that the dynamics (5) can be transformed to a convenient linear time-invariant form by exploiting transformations (6), (7), and (8). The proof of the lemma is omitted as it follows from straightforward (even though lengthy) mathematical derivations.

Lemma 1: Consider matrices in (6), (7), and (8). Then the following operation:

$$
\widehat{\xi}=R(\nu) \xi:=S(\nu) C(\nu) T(\nu) \xi,
$$

is a linear time-varying coordinates change, namely $R(\nu)$ is invertible for all $\nu$ and $R$ and $R^{-1}$ are uniformly bounded. Moreover, $R$ is periodic and transforms $A(\nu)$ into the following time-invariant form: $\widehat{A}=R^{\prime}(\nu) R^{-1}(\nu)+$ $R(\nu) A(\nu) R^{-1}(\nu)=\left[\begin{array}{ll|llll}0 & 0 & 0 & 0 & 0 & 0 \\ 0 & 0 & 0 & 0 & 0 & 0 \\ \hline 0 & 0 & 0 & 0 & 0 & 0 \\ 0 & 0 & 0 & 0 & 0 & 0 \\ 0 & 0 & 0 & 0 & 0 & 0 \\ 0 & 0 & 0 & 0 & 0 & 0 \\ 0 & 0 & 0\end{array}\right]$.

From the peculiar structure (quasi Jordan form) of the dynamic matrix $\hat{A}$, it is observed (as already emphasized for similar coordinate changes in [4]), that any periodic free motion of the original dynamics (1) can be suitably parametrized by specific selections of the state $\widehat{\xi}$ with the last component being zero. It is therefore convenient representing the system in terms of the error with respect to a desired motion $\xi^{r e f}$ :

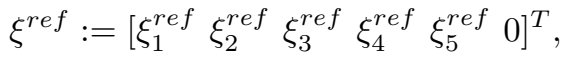

so that one may analyze the dynamics of the mismatch $\tilde{\xi}=$ $\widehat{\xi}-\xi^{r e f}$ between the coordinate $\widehat{\xi}$ in (10) and a constant reference value in (11), representing a desired target periodic motion.

\section{IMPULSIVE CONTROL OF THE RELATIVE DYNAMICS}

The coordinate transformation presented in Lemma 1 of the previous section is a useful means for suitably designing an impulsive control law assigning the firing times $t_{k}$ in (2) and also the corresponding selections of $\Delta v\left(t_{k}\right)$. Among others, one objective is to reduce as much as possible the fuel consumption well characterized in [10] when firing is achieved by 6 identical thrusters rigidly mounted to the satellite, and corresponding to the cost function $J=\sum_{t_{k} \in \mathcal{T}}\left|\Delta v_{x}\left(t_{k}\right)\right|+\left|\Delta v_{y}\left(t_{k}\right)\right|+\left|\Delta v_{z}\left(t_{k}\right)\right|=$ $\sum_{t_{k} \in \mathcal{T}}\left|\Delta v\left(t_{k}\right)\right|_{1}$, where $\mathcal{T}$ is the set of the times when impulsive thrusts are applied and $|\cdot|_{1}$ denotes the 1-norm. A more convenient expression of $J$ is given in terms of the representation in (5) and corresponds to:

$$
J=\sum_{\nu_{k} \in \mathcal{V}}\left|u\left(\nu_{k}\right)\right|_{1},
$$




$$
\widehat{B}(\nu)=\frac{1}{k^{2} \rho^{2}\left(1-e^{2}\right)}\left[\begin{array}{ccc}
0 & -\left(1-e^{2}\right) \rho s_{\nu} & 0 \\
0 & \left(1-e^{2}\right) \rho c_{\nu} & 0 \\
-e(1+\rho) \rho s_{\nu}-\frac{3 \sigma \rho^{3}}{\left(1-e^{2}\right)^{3 / 2}} & 0 & \frac{3 \sigma e \rho^{2} s_{\nu}}{\left(1-e^{2}\right)^{3 / 2}}-\rho^{3}+\rho^{2}+2 \rho \\
\left(1-e^{2}\right)(1+\rho) \rho s_{\nu} & 0 & \left(1-e^{2}\right) \rho^{2} c_{\nu} \\
(1+\rho) \rho c_{\nu}+e \rho & 0 & -\rho^{2} s_{\nu} \\
-3 \rho^{3} & 0 & 3 e \rho^{2} s_{\nu}
\end{array}\right]
$$

with $\mathcal{V}$ being the set of firing instants. Based on (13) we may formulate our control design problem as follows:

Problem 1: Given plant (1) and its equivalent form (5), design a state feedback impulsive control law selecting the firing instants $\nu_{k}, k \in \mathbb{N}$ and the corresponding inputs $u\left(\nu_{k}\right)$ such that

(i) for any selection of reference (11), the point $\left\{\xi=\xi^{r e f}\right\}$ is globally asymptotically stable for the closed loop dynamics;

(ii) the cost $J$ is minimized over a family of possible input selections.

To solve Problem 1, in this section we will propose hybrid control laws relying on the presence of a timer $\tau$ in charge of the sequencing of the impulsive control actions. Then, using the hybrid systems notation in [6] and state $\tilde{\xi}=\widehat{\xi}-\xi^{\text {ref }}$, we may write the following general dynamic description of the closed loop, enjoying the desirable property that timers $\nu$ and $\tau$ evolve in the compact set $[0,2 \pi]$ and that the flow equation for $\tilde{\xi}$ is $\tilde{\xi}^{\prime}=\widehat{A} \widehat{\xi}$, because $\widehat{A} \xi^{r e f}=0$ :

$$
\begin{aligned}
& \left\{\begin{array}{lr}
\tilde{\xi}^{\prime}=\widehat{A} \tilde{\xi}, & (\tilde{\xi}, \nu, \tau) \in \mathcal{C}, \\
\nu^{\prime}=1, & (\tilde{\xi}, \nu, \tau) \in \mathcal{D}_{\nu},
\end{array}\right. \\
& \begin{cases}\tilde{\xi}^{+}=\tilde{\xi}, \\
\nu^{+}=0, \\
\tau^{+}=\tau,\end{cases} \\
& \left\{\begin{array}{l}
\tilde{\xi}+=\tilde{\xi}+\widehat{B}(\nu) \gamma_{u}(\tilde{\xi}, \nu), \\
\nu^{+}=\nu, \\
\tau^{+}=\gamma_{\tau}(\tilde{\xi}, \nu),
\end{array}\right.
\end{aligned}
$$

In equation (14), the impulsive control law has been selected as a feedback controller:

$$
u=\gamma_{u}(\tilde{\xi}, \nu), \tau^{+}=\gamma_{\tau}(\tilde{\xi}, u) .
$$

Moreover, according to the coordinate change given in (10), and to the results of Lemma 1 , matrix $B(\nu)=$ $R(\nu) B(\nu)$, is a periodic function of $\nu$ arising from combining the similarity transformation in (10) with the input matrix in (5), and corresponds to the matrix reported in (12) at the top of this page.

Equation (14) is a compact representation of the impulsive feedback control action as a set of dynamical constraints that solutions should satisfy for their correct evolution. In particular, using an overall state $\eta=(\tilde{\xi}, \nu, \tau)$, this dynamics falls into the general class of systems studied in [6]:

$$
\begin{array}{ll}
\eta \in \mathcal{C}, & \dot{\eta}=F(\eta), \\
\eta \in \mathcal{D}, & \eta^{+} \in G(\eta) .
\end{array}
$$

For our model, the following selections are made:

$$
\begin{aligned}
\mathcal{D}_{\nu} & :=\mathbb{R}^{6} \times\{2 \pi\} \times[0,2 \pi], \\
\mathcal{D}_{u} & :=\mathbb{R}^{6} \times[0,2 \pi] \times\{0\}, \\
\mathcal{D} & :=\mathcal{D}_{\nu} \cup \mathcal{D}_{u}, \\
\mathcal{C} & :=\frac{\left.\mathbb{R}^{6} \times[0,2 \pi] \times[0,2 \pi]\right) \backslash \mathcal{D}}{},
\end{aligned}
$$

which, due to $(17 \mathrm{c})$, is a choice that prioritizes jumps. In particular, based on (14), functions $F$ and $G$ in (16) are selected as:

$$
\begin{aligned}
& F(\eta)=\left[\begin{array}{c}
\widehat{A} \tilde{\xi} \\
1 \\
-1
\end{array}\right] ; G(\eta)=\underset{i \in\{u, \nu\}}{\bigcup} \text { s.t. } \eta \in \mathcal{D}_{i} \\
& G_{i}(\eta) ; \\
& G_{\nu}(\eta)=\left[\begin{array}{c}
\tilde{\xi} \\
0 \\
\tau
\end{array}\right] ; G_{u}(\eta)=\left[\begin{array}{c}
\tilde{\xi}+\widehat{B}(\nu) \gamma_{u}(\tilde{\xi}, \nu) \\
\nu \\
\gamma_{\tau}(\tilde{\xi}, \nu)
\end{array}\right] .
\end{aligned}
$$

\section{Control laws}

In this section, we propose three different selections for the impulsive control law (15) solving Problem 1. These selections are different in terms of performance trade-offs and are comparatively illustrated on the example studies of Section V.

\section{A. Periodic norm-minimizing control}

While formulation (14), (15) is general enough to allow for aperiodic optimized sampling, the simplest possible selection of function $\gamma_{\tau}$ in (15) is given by periodic thrusters firing, corresponding to a certain period $\bar{\nu} \in[0,2 \pi]$ fixed a priori. This is equivalent to the following constant selection:

$$
\gamma_{\tau}(\tilde{\xi}, \nu)=\bar{\nu},
$$

encoding the fact that each pair of consecutive jumps has a fixed angular distance of $\bar{\nu}$.

Regarding the selection of the stabilizer $\gamma_{u}$, to be evaluated periodically, we make here a conservative selection leading to the useful feature that after each impulse, the state $\tilde{\xi}_{6}=\xi_{6}$ is driven to zero, so that in the absence of noise the spacecraft evolves through periodic (therefore bounded) motions. In particular, the following optimal selection is chosen:

$$
\begin{aligned}
& u^{\star}=\underset{u}{\arg \min }\left|\tilde{\xi}^{+}\right|^{2}, \text { subject to: } \\
& \quad \tilde{\xi}^{+}=\tilde{\xi}+\widehat{B}(\nu) u, \quad \tilde{\xi}_{6}^{+}=0 .
\end{aligned}
$$

Due to the specific structure of matrix function $\widehat{B}$ in (12) at the top of the page, we may provide an explicit form of 
the minimizer in (20) after defining the following quantities:

$$
\hat{b}_{6}(\nu)=\frac{1}{k^{2}}\left[\begin{array}{c}
\frac{3 \rho}{e^{2}-1} \\
0 \\
\frac{3 e \sin (\nu)}{1-e^{2}}
\end{array}\right], \hat{B}_{6}^{\perp}(\nu)=\left[\begin{array}{cc}
e \sin (\nu) & 0 \\
0 & 1 \\
\rho(\nu) & 0
\end{array}\right],
$$

which clearly satisfy $\hat{b}_{6}(\nu)^{T} \hat{B}_{6}^{\perp}(\nu)=0$ because matrix $\hat{B}_{6}^{\perp}(\nu)$ generates the orthogonal complement of $\hat{b}_{6}(\nu)$.

With these definitions in place, we may write the explicit expression of the proposed control law as:

$$
\begin{gathered}
\gamma_{u}(\tilde{\xi}, \nu)=u_{6}-\hat{B}_{6}^{\perp}(\nu)\left(\widehat{B}(\nu) \hat{B}_{6}^{\perp}(\nu)\right)^{-L}\left(\tilde{\xi}+\widehat{B}(\nu) u_{6}\right) \\
\text { with } u_{6}=-\frac{\hat{b}_{6}(\nu)}{\left|\hat{b}_{6}(\nu)\right|^{2}} \tilde{\xi}_{6},
\end{gathered}
$$

where $M^{-L}=\left(M^{T} M\right)^{-1} M^{T}$ denotes the left pseudoinverse of matrix $M$. The effectiveness of selection (21b) is stated in the next proposition.

Proposition 1: For any value of $\nu$, the inverses in function (21) always exist and selection (21) coincides with the minimizer in (20), namely $\gamma_{u}(\tilde{\xi}, \nu)=u^{\star}$.

Remark 1: Based on Proposition 1, a desirable property of control law (19), (21) is that it instantaneously minimizes the norm of $\tilde{\xi}$ constrained to the fact that the subsequent motion be periodic. Since the norm of $\hat{b}_{6}$ in (21a) is never zero, then clearly, equation (21b) is always well-posed and ensures that $\tilde{\xi}_{6}^{+}=0$. In addition to this, instantaneously minimizing the norm of $\tilde{\xi}$ also ensures the best possible decrease at the specific fixed instant of time enforced by the rigid periodic selection. With this logic in place, we can guarantee stability of the closed-loop but not convergence. Indeed, we can guarantee non-increase of $|\tilde{\xi}|$ across jumps but there is no guarantee of obtaining a strict decrease. As a result, we anticipate a slow convergence (if any) in our simulation section when using this controller. Despite this fact, the choice (19), (21) is still an interesting one because it ensures that approaching between the two satellites is performed through periodic (bounded) motions, leading to some degree of fault tolerance (in case of malfunctioning, the satellite is on a stable orbit).

It can be proven that the proposed controller solves part of item (i) of Problem 1. See [2, Thm 1] for details.

\section{B. Periodic bi-impulsive control}

A second selection that we propose for the controller in (15) is once again periodic, thereby corresponding to selection (19) for $\gamma_{\tau}$. However, it corresponds to a wiser selection of $\gamma_{u}$ (in terms of envisioned fuel consumption), performed in similar ways to what is proposed in [4], by focusing on the overall effect on the state $\tilde{\xi}$ of two impulses performed at a distance of $\bar{\nu}$ from one another. In particular, using straightforward computations, if two impulses $u_{1}$ and $u_{2}$ happen at times $\mu_{1}$ and $\mu_{2}=\mu_{1}+\bar{\nu}$, we obtain, along the corresponding solution:

$$
\Phi(-\bar{\nu}) \tilde{\xi}\left(\mu_{2}, j+2\right)=\tilde{\xi}\left(\mu_{1}, j\right)+\underbrace{\left[\hat{B}\left(\nu_{1}\right) \Phi(-\bar{\nu}) \hat{B}\left(\nu_{1}+\bar{\nu}\right)\right]}_{M\left(\nu_{1}, \bar{\nu}\right):=}\left[\begin{array}{l}
u_{1} \\
u_{2}
\end{array}\right]
$$

where $\mu_{1}, \mu_{2}$ denote the (angular) times, $j$ denotes the number of elapsed thruster firings, $\Phi(\mu)=e^{\widehat{A} \mu}=$ $\left[\begin{array}{cccccc}1 & 0 & 0 & 0 & 0 & 0 \\ 0 & 1 & 0 & 0 & 0 & 0 \\ 0 & 0 & 1 & 0 & 0 & \mu\left(1-e^{2}\right)^{-3 / 2} \\ 0 & 0 & 0 & 1 & 0 & 0 \\ 0 & 0 & 0 & 0 & 1 & 0 \\ 0 & 0 & 0 & 0 & 0 & 1\end{array}\right]$

is the state transition matrix of the

(LTI) flow dynamics in (14), and $\nu_{1}=\nu\left(\mu_{1}, j\right)$.

Based on relation (22), and to the end of selecting $u_{1}, u_{2}$ in such a way that $\tilde{\xi}\left(\mu_{2}, j+2\right)$ is zero, it is important to study the invertibility properties of matrix $M(\nu, \bar{\nu})$, which is done in the following conjecture. The result of the conjecture restricts the set of possible selections of $\bar{\nu}$ in (19).

Conjecture 1: For any value of $\nu \in[0,2 \pi]$, matrix $M(\nu, \bar{\nu})$ in (22) is invertible if and only if $\bar{\nu} \neq k \pi, k \in \mathbb{Z}$.

Conjecture 1 is motivated by numerical experience and its formal proof is regarded as future work. If Conjecture 1 holds, for any selection $\bar{\nu} \in(0,2 \pi) \backslash\{\pi\}$, equation (22) can be inverted to compute the unique pair of inputs $u_{1}^{\star}, u_{2}^{\star}$ ensuring $\tilde{\xi}\left(\mu_{2}, j+2\right)=0$ (namely that the state $\tilde{\xi}$ is driven to zero after two impulses separated by $\bar{\nu}$ times). Then, following a receding horizon type of paradigm, we may apply the first pulse and re-evaluate the control law at the next pulse. The above control design paradigm leads to the following selection:

$$
\gamma_{u}(\tilde{\xi}, \nu)=-\left[\begin{array}{ll}
I & 0
\end{array}\right] M(\nu, \bar{\nu})^{-1} \tilde{\xi} .
$$

Then, the overall control strategy (19), (23) guarantees item (i) of Problem 1 as established formally in [2, Thm 2].

\section{Non-periodic bi-impulsive control}

With the goal of minimizing the fuel consumption, a main drawback of the strategies proposed in the two previous sections is the fact that the (angular) time elapsed between two consecutive impulses is fixed and equal to $\bar{\nu}$. This is indeed a direct consequence of the simplified selection (19). In this section, we propose a different control paradigm, corresponding to an increased computational complexity, where the bi-impulsive control paradigm in (23) is combined with an optimized selection of the waiting time $\tau^{+}$before the next impulse. In particular, the overall control law corresponds to:

$$
\begin{aligned}
& \gamma_{\tau}(\tilde{\xi}, \nu)=\underset{\bar{\nu} \in[0,2 \pi]}{\arg \min }\left|M(\nu, \bar{\nu})^{-1} \tilde{\xi}\right|_{1}=\underset{\bar{\nu} \in[0,2 \pi]}{\arg \min } J, \\
& \gamma_{u}(\tilde{\xi}, \nu)=-\left[\begin{array}{ll}
I & 0
\end{array}\right] M\left(\nu, \gamma_{\tau}(\tilde{\xi}, \nu)\right)^{-1} \tilde{\xi} .
\end{aligned}
$$

The new control law (24) corresponds to selecting an optimal elapsed time $\mu$ before the next impulse, according to the fuel consumption index (13). In particular, the corresponding optimization is nonlinear and, because of Conjecture 1, it corresponds to minimizing the argument in the two intervals $(0, \pi)$ and $(\pi, 2 \pi)$ where matrix $M(\nu, \cdot)$ is invertible. Then, the minimum can be selected as the minimum of the two minimizers.

Similar to the previous solution (19), (23), it can be formally established that controller (24) solves Problem 1. See [2, Thm 2] for details.

\section{Simulations}

In this section, we present the simulation results obtained with the control laws designed in Section IV and system (14) (equivalently (16)-(18)) using a linearized model simulator. In these simulations, an orbit with a semi-major axis of $7011 \mathrm{~km}$ and eccentricity $e=0.4$ will be considered for the leader vehicle. The simulations aim at stabilizing a periodic trajectory specified by a suitable selection of $\xi^{\text {ref }}$ 
in (11), which is free to evolve inside a tolerance box $\mathcal{B}$. The tolerance box $\mathcal{B}$ is centered at point $\zeta_{f}$ (expressed in the target's position frame used in (1)) and has positive and negative widths $\zeta_{t o l}$ in the three LVLH directions, where $\zeta_{f}=\left[\begin{array}{lll}100 & 0 & 0\end{array}\right]^{T} \mathrm{~m}$ and $\zeta_{t o l}=\left[\begin{array}{lll}150 & 25 & 25\end{array}\right]^{T} \mathrm{~m}$.

In [3], a method is introduced to obtain the value of $\xi^{r e f}$ in (11) ensuring that the corresponding periodic evolution in the LVLH coordinates is always contained in the tolerance box $\mathcal{B}$. The resulting values of the reference state $\xi^{\text {ref }}$ correspond to:

$$
\xi^{r e f}=\left[\begin{array}{llllll}
7.68 & 17.68 & 87.78 & 33.04 & -15.77 & 0
\end{array}\right]^{T},
$$

where we emphasize that the last element is zero (a necessary and sufficient condition for periodic motion).

For each one of the three control laws in Section IV, four different initial conditions $\zeta_{0}$ for state $\zeta$ in (1) have been used, selected as:

$$
\begin{aligned}
\zeta_{01} & =\left[\begin{array}{llllll}
500 & 400 & 10 & 0 & 0 & 0
\end{array}\right]^{T}, \\
\zeta_{02} & =\left[\begin{array}{llllll}
-200 & 100 & 200 & 0 & 0 & 0
\end{array}\right]^{T}, \\
\zeta_{03} & =\left[\begin{array}{llllll}
100 & -350 & -20 & 0 & 0 & 0
\end{array}\right]^{T}, \\
\zeta_{04} & =\left[\begin{array}{llllll}
320 & 0 & -64 & 0 & 0 & 0
\end{array}\right]^{T},
\end{aligned}
$$

where in (26) the first three components are meters and the last three are meters per second. The initial relative velocity has been selected to be zero to account for the fact that the starting point of our trajectory may be a holding point arising from a previous station keeping along the space mission. Note that $\zeta_{04}$ is chosen to lie in the orbital plane of the target (the second component is zero).

The four initial conditions $\zeta_{01}, \zeta_{02}, \zeta_{03}$ and $\zeta_{04}$ in (26) correspond to four different initial distances from the tolerance box $\mathcal{B}$ :

$$
\begin{aligned}
\left|\zeta_{01}\right|_{\mathcal{B}}=512.9571 \mathrm{~m} & \left|\zeta_{02}\right|_{\mathcal{B}}=314.2451 \mathrm{~m} \\
\left|\zeta_{03}\right|_{\mathcal{B}}=325.0000 \mathrm{~m} & \left|\zeta_{04}\right|_{\mathcal{B}}=326.3372 \mathrm{~m}
\end{aligned}
$$

with the distance from the bounding box $\mathcal{B}$ being defined as: $|\zeta|_{\mathcal{B}}=\left|\mathrm{dz}_{\zeta_{\text {tol }}}\left(\zeta_{1 . .3}-\zeta_{f}\right)\right|$, where $\zeta_{1 . .3}=(x, y, z)$ contains the first three components of state $\zeta$ in (1) and $\mathrm{dz}_{\zeta_{\text {tol }}}(x)$ is the decentralized vector dead-zone function with limits $\zeta_{t o l}$.

The initial leader true anomaly values $\nu_{0}$ vary from 0 to 360 degrees in equally spaced intervals of 10 degrees. All our simulations have been performed from the initial true anomaly $\nu_{0}$ up to a final true anomaly $\nu_{f}=\nu_{0}+20 \pi$, namely ten orbital periods later.

Two performance indexes are considered: the fuel consumption $J$ in (13) and the true anomaly $\nu_{B}$ or angular time in terms of orbital periods (time elapsed when the follower first reaches the bounding box $\mathcal{B}$ ). Figures 1 and 2 represent respectively these two indexes fixing each one of the initial conditions in (26) and using the three control laws in Section IV, where:

- the orange line $(-\times-)$ refers to controller (19), (21) of Section IV-A, with the selection $\bar{\nu}=\pi / 2$ in (19);

- the blue line $(-\circ-)$ refers to controller (19), (23) of Section IV-B, with the same firing interval $\bar{\nu}=\pi / 2$;

- the green line $(-\triangleleft-)$ refers to the response using controller (24) discussed in Section IV-C.

Figure 1 shows the cost $J$ for each control law when the leader's initial true anomaly $\nu_{0}$ varies. From this figure it is possible so see that for every initial state $\zeta_{0}$ and every control law, the minimum cost always appears in the proximities of 180 (deg), corresponding to the apogee of the orbit. It would be interesting therefore to perform the satellite maneuver when the leader's initial true anomaly $\nu_{0}$ lies in the neighborhood of this value in order to minimize the amount of dispensed fuel. As expected, controller (24) always leads to the minimum value of $J$.
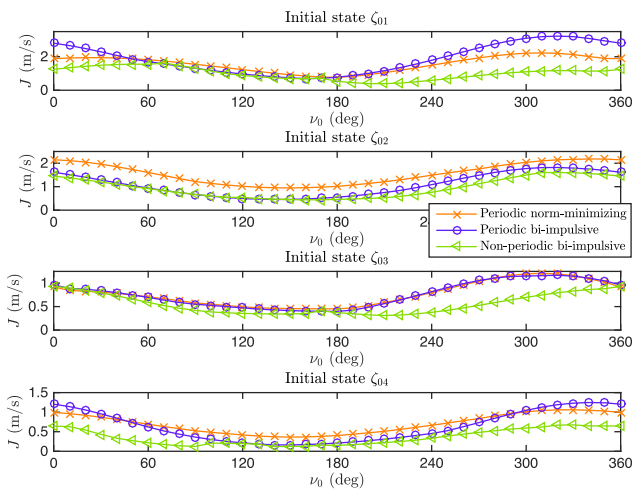

Fig. 1: Comparison of the cost $J$ among the three control laws for the initial states in (26).

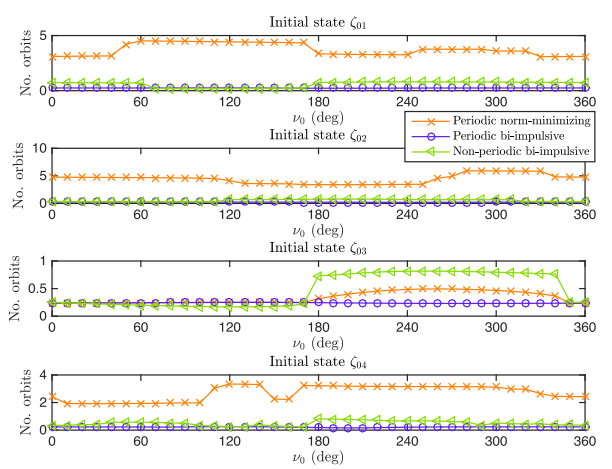

Fig. 2: Comparison of the number of orbital periods $\nu_{B} / 2 \pi$ among the three control laws for the initial states in (26).

\begin{tabular}{ccccc}
\hline$\zeta_{0}$ & $\begin{array}{c}\text { Min. } \\
\text { value }\end{array}$ & $\begin{array}{c}\text { Periodic norm- } \\
\text { minimizing }\end{array}$ & $\begin{array}{c}\text { Periodic } \\
\text { bi-impulsive }\end{array}$ & $\begin{array}{c}\text { Non-periodic } \\
\text { bi-impulsive }\end{array}$ \\
\hline$\zeta_{01}$ & $J$ & 0.7722 & 0.6942 & 0.3942 \\
& $\nu_{B}$ & 3.0904 & 0.2230 & 0.1849 \\
\hline$\zeta_{02}$ & $J$ & 0.9448 & 0.4566 & 0.4402 \\
& $\nu_{B}$ & 3.4036 & 0.2013 & 0.2817 \\
\hline \multirow{2}{*}{$\zeta_{03}$} & $J$ & 0.4522 & 0.3991 & 0.3108 \\
& $\nu_{B}$ & 0.2349 & 0.2306 & 0.1690 \\
\hline \multirow{2}{*}{$\zeta_{04}$} & $J$ & 0.3634 & 0.1612 & 0.1188 \\
& $\nu_{B}$ & 1.9181 & 0.1592 & 0.2269 \\
\hline
\end{tabular}

TABLE I: Minimum values of fuel consumption $J(\mathrm{~m} / \mathrm{s})$ and angular time $\nu_{\mathcal{B}}$ (no. of orbits) for all the simulations presented in Figures 1 and 2.

All Figures from 1 to 6 highlight that the speed of convergence to the bounding box $\mathcal{B}$ is relatively slow when using the control law of Section IV-A. In fact, from Table I, it is possible to observe that parameter $\nu_{\mathcal{B}}$ is much larger for this control law than the one obtained in the two other cases. Figures 3, 4, 5 and 6 represent the trajectories of the follower satellite for an initial leader true anomaly of 180 
(deg), and it can be seen that the trajectories corresponding to the first control law are much longer than the ones of the two other controls.

The periodic norm-minimizing control presents a slow convergence, while associated to an increased fuel consumption. On the other hand, an advantage of this controller is that the solution evolves along periodic motions, thereby being fault tolerant. Instead, the two bi-impulsive solutions show a reduced fuel consumption with a strong preference for the non-periodic one, always leading to a more than substantial fuel saving.

Both the bi-impulsive control laws discussed in Sections IV-B and IV-C behave better than the previous one in terms of convergence speed. In fact the reference trajectory is tracked after the firing period $\bar{\nu}$ with zero error, by design.

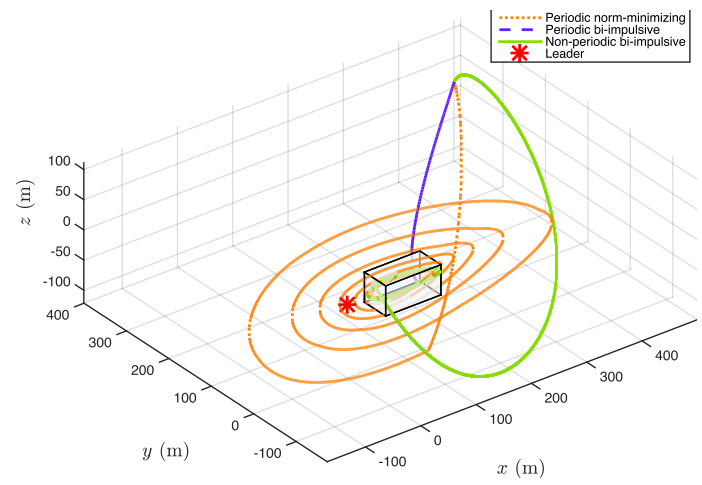

Fig. 3: Evolution of $\zeta_{1 . .3}$ with the three control laws from the initial state $\zeta_{01}$ and $\nu_{0}=180^{\circ}$.

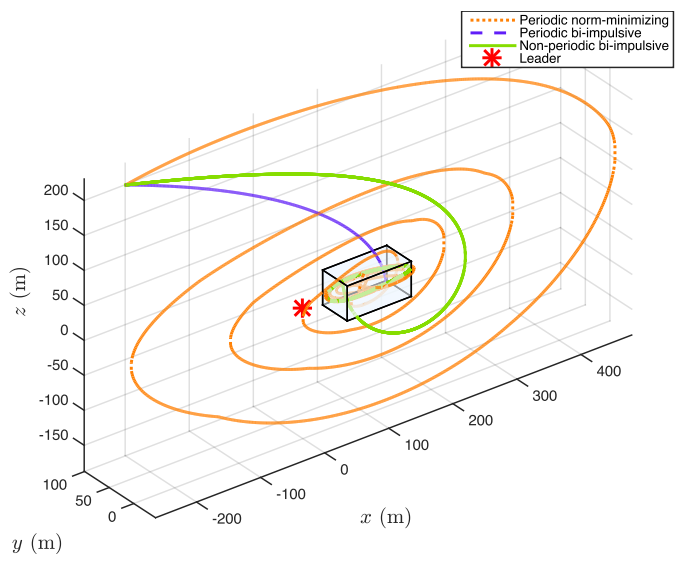

Fig. 4: Evolution of $\zeta_{1 . .3}$ with the three control laws from the initial state $\zeta_{02}$ and $\nu_{0}=180^{\circ}$.

\section{CONCLUSIONS}

In this article, a new model, based on Floquet-Lyapunov theory, is developed in order to obtain a linear time-invariant free motion representation of the rendezvous problem. This problem is then recast as a stabilization problem for a periodic trajectory in a hybrid dynamical system framework. Two different control laws are developed and compared to a control scheme given in [4], which is re-interpreted in this hybrid context.

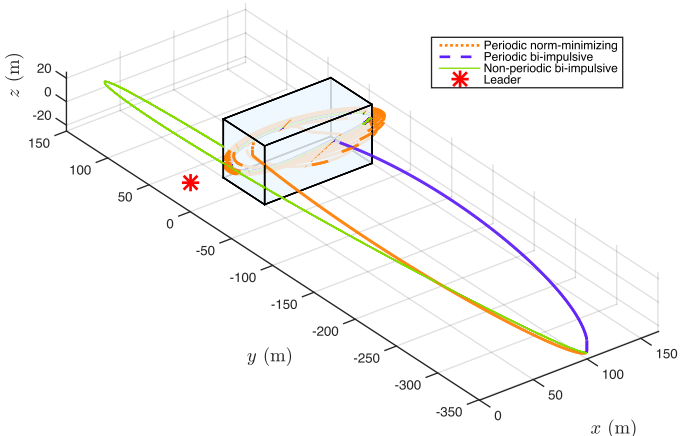

Fig. 5: Evolution of $\zeta_{1 . .3}$ with the three control laws from the initial state $\zeta_{03}$ and $\nu_{0}=180^{\circ}$.

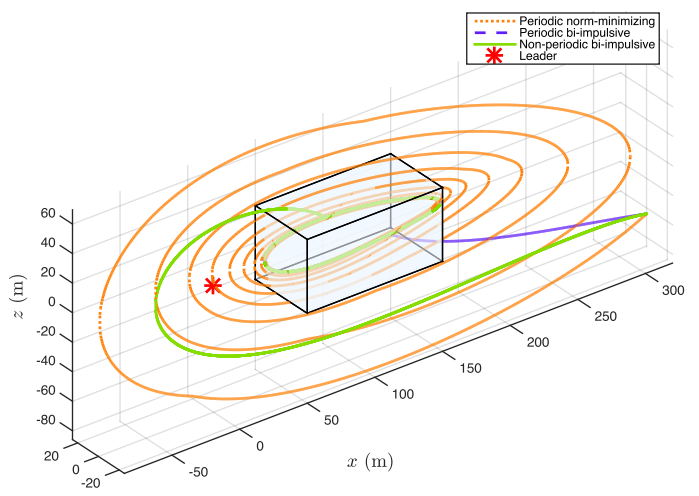

Fig. 6: Evolution of $\zeta_{1 . .3}$ with the three control laws from the initial state $\zeta_{04}$ and $\nu_{0}=180^{\circ}$.

Alternative trade-offs should be investigated that adjust $\bar{\nu}$ within optimized fuel consumption under some constraints on the maximum distance reached during the transient.

\section{REFERENCES}

[1] K.T. Alfriend and H. Schaub. Dynamics and control of spacecraft formations: Challenges and some solutions. Journal of the Astronautical Sciences, 48(2):249-267, 2000.

[2] Mirko Brentari, Denis Arzelier, Christophe Louembet, Sofia Urbina and Luca Zaccarian. A hybrid control framework for impulsive control of satellite rendezvous. working paper or preprint, September 2015.

[3] G. Deaconu, C. Louembet, and A. Theron. Constrained periodic spacecraft relative motion using non-negative polynomials. In American Control Conference (ACC), 2012, pages 6715-6720, June 2012.

[4] G. Deaconu, C. Louembet, and A. Theron. A two-impulse method for stabilizing the spacecraft relative motion with respect to a periodic trajectory. In Decision and Control (CDC), 2012 IEEE 51st Annual Conference on, pages 6541-6546, Dec 2012.

[5] W. Fehse, editor. Automated rendezvous and docking of spacecraft. Cambridge Aerospace Series. Cambridge University Press, Cambridge, UK, 2003.

[6] R. Goebel, R.G. Sanfelice, and A.R. Teel. Hybrid Dynamical Systems: modeling, stability, and robustness. Princeton University Press, 2012.

[7] P. Gurfil. Relative motion between elliptic orbits: Generalized boundedness conditions and optimal formationkeeping. Journal of Guidance, Control and Dynamics, 28(4):761-767, July 2005.

[8] G. Inalhan, M. Tillerson, and J.P. How. Relative dynamics and control of spacecraft formations in eccentric orbits. Journal of Guidance, Control and Dynamics, 25(1):48-59, January-February 2002.

[9] C. Louembet, D. Arzelier, and G. Deaconu. Robust rendezvous planning under maneuver execution errors. Journal of Guidance, Control, and Dynamics, 2015.

[10] I.M. Ross. 6 space trajectory optimization and 11 -optimal control problems. Elsevier Astrodynamics Series, 1:155-VIII, 2007.

[11] H. Schaub and J.L. Junkins. Analytical mechanics of space systems. Education Series. AIAA, Reston, Virginia, USA, 2003.

[12] J. Tschauner. The elliptic orbit rendezvous. In AIAA 4th Aerospace Sciences Meeting, Los Angeles, Californie, USA, Juin 1966. 